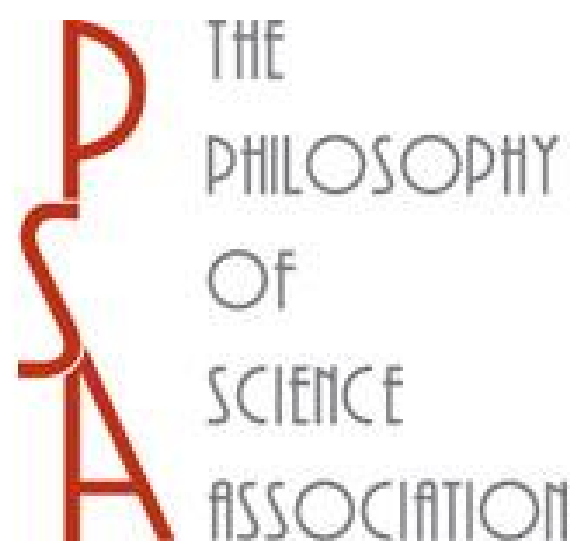

Reconsidering the Concept of Equilibrium in Classical Statistical Mechanics Author(s): Janneke van Lith

Source: Philosophy of Science, Vol. 66, Supplement. Proceedings of the 1998 Biennial Meetings of the Philosophy of Science Association. Part I: Contributed Papers (Sep., 1999), pp. S107-S118

Published by: The University of Chicago Press on behalf of the Philosophy of Science Association

Stable URL: http://www.jstor.org/stable/188765

Accessed: 07-12-2016 09:35 UTC

JSTOR is a not-for-profit service that helps scholars, researchers, and students discover, use, and build upon a wide range of content in a trusted digital archive. We use information technology and tools to increase productivity and facilitate new forms of scholarship. For more information about JSTOR, please contact support@jstor.org.

Your use of the JSTOR archive indicates your acceptance of the Terms \& Conditions of Use, available at http://about.jstor.org/terms

The University of Chicago Press, Philosophy of Science Association are collaborating with JSTOR to digitize, preserve and extend access to Philosophy of Science 


\title{
Reconsidering the Concept of Equilibrium in Classical Statistical Mechanics
}

\author{
Janneke van Lith ${ }^{\dagger}$ \\ Utrecht University
}

\begin{abstract}
In the usual procedure of deriving equilibrium thermodynamics from classical statistical mechanics, Gibbsian fine-grained entropy is taken as the analogue of thermodynamical entropy. However, it is well known that the fine-grained entropy remains constant under the Hamiltonian flow. In this paper it is argued that we need not search for alternatives for fine-grained entropy, nor do we have to reject Hamiltonian dynamics, in order to solve the problem of the constancy of fine-grained entropy and, more generally, to account for the non-equilibrium part of the laws of thermodynamics. Rather, we have to weaken the requirement that equilibrium be identified with a stationary probability distribution.
\end{abstract}

1. Introduction. Consider a physical system in thermal equilibrium, which is then disturbed by outside influences. Think for example of a gas that is initially confined to a small part of a container, and at a certain time is allowed to diffuse into the whole available space when a barrier is removed. Thermodynamics predicts that there will be a new equilibrium after some time. Also, if there is no heat exchange with the environment, the entropy of the final state will be larger than that of the initial state. Can classical statistical mechanics reproduce these predictions? That is the main theme of this paper.

It is generally believed that classical statistical mechanics can reproduce all of thermodynamics, when we restrict ourselves to equilibrium

$\dagger$ Institute for History and Foundations of Mathematics and Science, Utrecht University, P.O. Box 80000, 3508 TA Utrecht, The Netherlands; e-mail: j.h.vanlith@ phys.uu.nl.

$\ddagger I$ would like to thank Dennis Dieks and Jos Uffink for valuable comments on earlier versions of this paper.

Philosophy of Science, 66 (Proceedings) pp. S107-S118. 0031-8248/99/66supp-0009\$0.00 Copyright 1999 by the Philosophy of Science Association. All rights reserved. 
properties. The way to proceed is as follows. We identify states of thermal equilibrium with probability distributions over the phase space that are constant in time. For the special cases of isolated systems and systems in thermal contact with a heat reservoir, we take the microcanonical and canonical probability distributions respectively. Further, we identify the so-called fine-grained entropy,

$$
S_{f g}=-k \int \rho(x) \ln \rho(x) d x
$$

with the thermodynamical entropy, where $\rho(x)$ denotes the probability density over phase space, and $k$ is Boltzmann's constant. If we introduce the expression for the (micro-)canonical distribution in (1), it turns out that the fine-grained entropy coincides with the thermodynamic entropy, up to an unimportant additive constant. It seems that we thus retrieve all thermodynamic relations by identifying $S_{f g}$ with entropy, and the Gibbsian ensembles as states of thermal equilibrium. The entropy change in the above mentioned process of sudden expansion of a gas can now be obtained from the fine-grained entropies of the initial and the final state.

However, it is well known that the fine-grained entropy stays constant under the action of a Hamiltonian flow, as a consequence of Liouville's theorem. That is, if, instead of identifying the final state of thermal equilibrium with a microcanonical distribution, we calculate the final distribution by applying Hamilton's equations to each point in phase space (i.e., apply Liouville's theorem to the initial probability distribution), then a distribution results with the same entropy as the initial distribution. Therefore, the final distribution cannot be the desired microcanonical one. The previously mentioned reproduction of thermodynamical results is inconsistent with the underlying dynamical evolution.

As Callender puts it:

The ensemble at the later time should be the Hamiltonian time development of the earlier ensemble; otherwise the statement that the system is governed by Hamilton's equations is a fiction. ... The time development of the earlier ensemble will not lead it to approach the later one in any way. This, I believe, is the real problem with fine-grained entropy, not that it doesn't increase between equilibrium states. $(1997,8)$

So, the real problem is that according to Hamilton's equations of motion the microcanonical distribution corresponding to the initial state cannot evolve into the microcanonical distribution corresponding to the final state. However, contrary to Callender's statement, the earlier 
ensemble typically does approach the later one in a relevant sense, and the approach I shall outline in this paper benefits from this fact. I want to pay special attention to this problem of reconciling the account of equilibrium with the Hamiltonian time evolution, as a part of the more general topic of reproducing the zeroth and second law.

A popular approach to this general topic is to replace the Hamiltonian dynamics by some other evolution. Then Liouville's theorem will in general not hold, and the above inconsistency disappears. Many approaches to thermal physics fall within this framework: e.g., approaches based on a Markov assumption, on master equations, the subdynamics of the Brussels school, and interventionism. ${ }^{1}$ However, adopting such a strategy immediately leads to an abandonment of the program of reducing thermodynamics to statistical mechanics, at least when the latter theory is construed as Hamiltonian dynamics for the system of interest, supplemented with probability considerations. In this paper I want to explore the possibilities of describing irreversible processes while remaining within this program.

Another approach is to replace the fine-grained entropy by the coarse-grained entropy $S_{c g}$, which need not be constant under the Hamiltonian flow (again, a discussion can be found in Sklar 1993). Thus, it escapes the paradox of the constant entropy; moreover, it can be shown that if the initial state is a uniform distribution, the coarse-grained entropy can only increase, indicating at least some agreement with the thermodynamical entropy. Whether these kinds of results suffice to show that $S_{c g}$ is a good analogue of the thermodynamical entropy is however yet to be seen. Also, there are well-known objections to the justification of the coarse-graining procedure, which is said to be subjective. Opponents of the coarse-graining approach argue that the existence of thermodynamic behavior cannot (solely) be due to lack of human capabilities to perform accurate measurements. Be that as it may, replacing the entropy function by a different one does not solve the previously mentioned inconsistency, since the inconsistency can be formulated without even mentioning entropy. Therefore I will not discuss the coarse-graining approach further.

In this paper I will propose a new approach, in which the conditions for equilibrium are relaxed. This redefinition also affects so-called quasistatic processes. Next, I will define entropy changes along quasistatic processes only. Then, entropy will equal the fine-grained entropy of the Gibbsian ensembles in equilibrium, but its time evolution will be different, in such a way that the inconsistency is circumvented $(\$ 5$ and 6). Finally, I will discuss briefly whether the more general goal of

1. See Sklar 1993 for a discussion of these approaches, and for references. 
reproducing the zeroth and second laws of thermodynamics can be achieved in my new approach (\$7). But I begin by taking a closer look at the theory of thermodynamics itself $(\$ 2)$, and the successful reproduction of it by statistical mechanics in the case of equilibrium ( $\$ 3)$.

2. The Zeroth and Second Law of Thermodynamics. In thermodynamics a system is said to be in equilibrium iff all its observable characteristics are constant in time, when the system is left to itself. The orthodox theory of thermodynamics deals mainly with such equilibrium situations. Still, at two major points in the theory a general statement about non-equilibrium processes is introduced, namely both in the zeroth and the second law. The former says that every isolated system will eventually reach an equilibrium state. The latter, in the form of Kelvin's principle, says that it is impossible to extract heat from a single heat reservoir and perform an equivalent amount of work in any cyclic process, without further effect. From this principle the law of increase of entropy is derived.

The central problem of this paper relates to both the approach to equilibrium and the increase of entropy. Remember our paradigm example of a gas that is suddenly allowed to diffuse into a container. The two claims of thermodynamics were that a new equilibrium will settle in, and that the entropy will increase.

Entropy in thermodynamics is a state function, i.e., a function of the macroscopic quantities that define the thermodynamic state of the system. It is formally introduced as the total differential $\delta S_{T D}=\delta Q / T$, where $\delta Q$ is to be evaluated along quasistatic processes, that is, processes that are so slow that the system can be considered to be in equilibrium all the time.

Outside equilibrium entropy is not defined. Still, it is possible to derive a general statement (the second law) about the change of entropy in all processes beginning and ending in equilibrium states. If these endpoints can also be connected by some quasistatic process, this entropy change obeys

$$
\Delta S_{T D} \geq \int \delta Q / T \text {. }
$$

For adiabatic processes $(\delta Q=0)$ this reduces to the more familiar statement that the entropy of a thermally isolated system can never decrease.

For our discussion two points need to be stressed. First, we have seen that thermodynamic entropy is only defined for equilibrium states. From this it follows immediately that the second law of thermodynamics does not, as is sometimes claimed, say that entropy increases mon- 
otonically during the approach to equilibrium. The only processes during which the entropy is defined all the time are quasistatic processes. ${ }^{2}$

Second, note that in order to get any change of entropy, some outside influence is necessary. A system in equilibrium will always remain in equilibrium, unless it is perturbed from the outside.

3. A Standard Account of Equilibrium Statistical Mechanics. We now turn to a discussion of the well-known procedure of deriving equilibrium thermodynamics from statistical mechanics; this will, as noted before, eventually lead to an inconsistency with the underlying dynamics. In order to make the connection between the two theories, identifications are made between thermodynamical and statistical mechanical concepts.

First of all, thermodynamic states are identified, not with points in phase space, but with probability distributions over them. An important special case is formed by the equilibrium states, which are identified with stationary probability distributions, the Gibbsian probability distributions (or ensembles) being the most important examples. Second, thermodynamic quantities are either identified with ensemble averages of phase functions (for instance energy and pressure), or with functionals on the probability distribution (notably entropy and temperature).

It is well known that the fine-grained entropy (1) of the standard Gibbs ensembles displays the appropriate thermodynamical behaviour in case of time-independent phenomena. For instance, when we introduce the canonical distribution function for the ideal gas in (1), we arrive at

$$
S_{f g}(T, V)=N k\left(\ln V+\frac{3}{2} \ln (2 \pi m k T)\right)+\text { const. }
$$

which is familiar from thermodynamics. In general, the entropy will be a function of the external parameters (like the volume $V$ ) and the parameters characterizing the ensemble (like the temperature $T$ in the canonical ensemble), and it will be the same function of those quantities as $S_{T D}$ is.

When Gibbs (1902) introduced the expression (1) for the finegrained entropy, he was reluctant to identify it with the thermodyn-

2. Ehrenfest-Afanassjewa (1956) introduces a useful distinction between quasistatic processes and quasi processes. The latter are curves in state space, consisting of consecutive equilibrium states. Therefore, they are not real processes. The former are real processes, which approximate quasi processes in the sense that they are extremely slow. The equality sign in (2) holds both for quasi processes and for quasistatic processes. 
amical entropy for general ensembles. But for the canonical ensembles we can actually derive it (see Uhlenbeck and Ford 1963, 21-24). That is, we can introduce entropy, just like in thermodynamics, as the total differential belonging to heat, which is defined as

$$
T \delta S=\delta Q \equiv \delta E-\delta W .
$$

Here the quantities on the right hand side are calculated as averages in the canonical ensemble: energy $(E)$ as that of the Hamiltonian, and work $\delta W$ as

$$
\delta W=\sum_{k}\left\langle\frac{\partial H}{\partial a_{k}}\right\rangle \delta a_{k},
$$

where the $a_{k}$ denote the external parameters, such as the volume. The temperature is connected to the parameter in the distribution function in the usual way, $T=1 / \mathrm{k} \beta$. Remember that in thermodynamics the left equation in (4) holds only for quasi and quasistatic processes. The crucial point is that these are now construed as processes in which the system is always considered to be canonically distributed. That is, it is assumed that after small changes in temperature or in the external parameters the system will again be member of a canonical ensemble, albeit a different one. In this respect the system is in equilibrium all the time during these processes.

Following these lines, we find an entropy of (grand-)canonically distributed systems which equals the fine-grained entropy of these ensembles. For the microcanonical ensemble the situation is a bit more difficult, since temperature is not a parameter of this distribution. However, after adding an appropriate definition of $\beta$ as a phase function, e.g., as the structure function $\omega(E, V, \ldots)$ divided by the integrated structure function $\Omega(E, V, \ldots)$, similar results apply here also. ${ }^{3}$ For the Gibbsian ensembles, this completes the standard account of equilibrium thermodynamics.

4. The Paradox of Constant Fine-Grained Entropy. A well-known problem arises however if we take a closer look at the time evolution of the

3. This definition amounts to choosing one of the sets of analogies already proposed by Gibbs $(1902,170)$. It seems that we put in the desired result by hand. But note that we at least gain the description of quasistatic processes, something that Gibbs himself does not offer. Also, our construction assures the thermodynamical relationship between entropy, temperature, and energy; a change in the definition of temperature leading to a change in entropy. Some arbitrariness remains, but that is also the case in thermodynamics itself. 
entropy. If the evolution of the fine-grained entropy is calculated by applying Hamilton's equations to every point $x$ in phase space, i.e.,

$$
S_{f g}(t)=-k \int \rho\left(T_{t} x\right) \ln \rho\left(T_{t} x\right) d x,
$$

then $S_{f g}$ is constant as a result of Liouville's equation, for an arbitrary distribution $\rho$. Therefore, describing time-dependent thermodynamical phenomena (notably the implications of the second law) seems impossible with the fine-grained entropy as the analogue of thermodynamical entropy.

On the other hand, in the above derivation of the entropy of the Gibbsian ensembles, entropy does change during "infinitely slow" processes. Moreover, at any stage during these processes, the entropy equals $S_{f g}$.

The seeming contradiction can be resolved by noting that an evolution from one canonical distribution to another is dynamically forbidden. Therefore, the above processes consisting of consecutive canonical distributions are in fact in conflict with the dynamical evolution of the phase points (although they may be good approximations of dynamical processes). Thus, in the terminology of Ehrenfest-Afanassjewa, these processes are really analogous to quasi processes rather than to quasistatic processes (cf. fn. 2), because no real physical system can undergo such a process.

We therefore conclude that the standard account of equilibrium and "infinitely slow" processes is inconsistent with a Hamiltonian time evolution between equilibrium states, as was stated already in the introduction. Note that this inconsistency can be formulated without even mentioning entropy. Therefore its resolution cannot be expected from changing to an entropy function that may increase under the influence of the Hamiltonian evolution, such as the coarse-grained entropy.

The question is now whether a better analogue of quasistatic processes can be found, in such a way that they are allowed by the dynamical laws. In Sections 5 and 6, I will propose such an analogue. Then, defining entropy differences along these processes will result in an entropy function that may change during real, physical processes, thereby solving the paradox of the constant fine-grained entropy.

5. Equilibrium. In the previous sections we were led to the suggestion that the identification of thermal equilibrium with stationary distributions may be too strict. The time evolution of an arbitrary timedependent probability distribution will, strictly speaking, never lead to a stationary distribution. It might however be expected to approximate a stationary distribution, in some relevant sense. I therefore propose 
to relax this condition, and allow also approximately stationary distributions to represent equilibrium.

To be more specific, let us consider a class $\Omega$ of macroscopically relevant quantities and define the system to be in equilibrium from $t=\tau$ onwards iff its distribution $\rho_{t}$ obeys

$$
\forall F \in \Omega, \forall t \geq \tau, \exists c:\left|\langle F\rangle_{\rho_{t}}-c\right| \leq \varepsilon_{F} .
$$

That is, a system is in equilibrium when the ensemble averages of phase functions in some class $\Omega$ are time-independent, or may fluctuate in time at most within some small, fixed intervals $\varepsilon_{F}$. An advantage of this definition is that the reference to macroscopic quantities makes it close to the thermodynamical definition.

Of course this definition has to be made more precise. Some numbers $\varepsilon_{F}$ have to be fixed, the most obvious proposal being small fractions of the limiting values of the ensemble averages. Also it has to be specified what the relevant macroscopic quantities are. A straightforward choice is to take just the quantities that are used in the thermodynamical description of the phenomenon, but various other options are conceivable.

The main point I wish to argue for is that a characterization of equilibrium along these lines is compatible with a Hamiltonian evolution between equilibrium states. Indeed, irrespective of the choice of the class $\Omega$ and of $\varepsilon_{F}$, dynamical evolution from one equilibrium state to another is now possible, as will be demonstrated below for the special case of mixing systems. The previous argument showing the inconsistency is invalidated, even for systems for which we cannot actually prove they will approach equilibrium. Moreover, I would claim that in weakening the conditions for equilibrium we have not lost any desirable property of equilibrium. On the contrary, due to the reference to macroscopic quantities we have come closer to the thermodynamical concept.

An important result that is relevant for this proposal applies to socalled mixing systems, i.e., dynamical systems obeying

$$
\lim _{t \rightarrow \infty} \mu\left(T_{t} A \cap B\right)=\mu(A) \mu(B) \quad \forall A, B \subset \Gamma
$$

where $T_{t} A$ denotes the time evolution of the set $A$ and $\mu$ stands for the microcanonical measure. Suppose that such a system is described by an arbitrary, time-dependent probability density $\rho_{t}$ (thus, the probability measure is required to be absolutely continuous (a.c.) with respect to the microcanonical (Lebesgue) measure). Then all ensemble averages will, in the infinite time limit, coincide with the microcanonical averages: 


$$
\lim _{t \rightarrow \infty}\langle F\rangle_{\rho_{t}}=\langle F\rangle_{\mu}
$$

for every bounded, $\mu$-measurable function $F{ }^{4}$ In this sense every a.c. measure converges to the microcanonical measure, which is of course stationary in time. Note however that the fine-grained entropy remains constant and does not converge to the fine-grained entropy of the microcanonical ensemble.

On the basis of our previous association of thermodynamic equilibrium with time-independent probability distributions, we would be forced to conclude that a mixing system will not reach equilibrium, despite the fact that in the long run not a single ensemble average can be discriminated from the microcanonical average. That is, we have no (macroscopic) means to discriminate our system from one which would be regarded as being in equilibrium. With our new definition on the other hand, mixing systems will indeed reach equilibrium, because the microcanonical averages are of course constant in time. Note that it is not necessary for this conclusion to assume the mixing property. It suffices if we demand mixing only with respect to the class $\Omega .{ }^{5}$

6. Quasistatic Processes and Entropy. In the previous section, a new understanding of the statistical mechanical analogue to thermodynamic equilibrium was given, which makes dynamical transitions from one equilibrium state to another possible. A natural step to take next is to define the notion of quasistatic processes in statistical mechanics, in such a way that they are dynamically allowed as well. The final step is then to define entropy and temperature according to $T \delta S=\delta Q$ along quasistatic processes in this new understanding. It then follows immediately that entropy changes along processes that are dynamically allowed, thus solving the "paradox of the constancy of the fine-grained entropy."

In thermodynamics, a quasistatic process is a process that is driven by external interventions that are exercised "infinitely slowly." At any stage the system can be considered to be in (or very close to) equilibrium, although in the long run there will be observable changes.

As a result, if at any stage during a quasistatic process the external interventions were stopped, the system would be in equilibrium immediately, and stay there. I propose to use this fact as a definition of

4. In fact, for a.c. measures (8) and (9) are equivalent conditions. For a proof, see Mackey 1993, 63.

5. That is, we require relation (9) to hold only for all $F \in \Omega$. Interestingly, Earman and Rédei (1996) come to the same requirement in their discussion of why equilibrium statistical mechanics works in predicting observed values of macroscopic quantities. 
quasistatic processes in statistical mechanics, where of course equilibrium should be understood in the sense of the definition in the previous section. Thus, a quasistatic processes is a very slow process such that, if the external interventions were switched off at a certain time, the ensemble averages of all functions $F \in \Omega$ from that time on would not diverge more than $\varepsilon_{F}$ from a constant value. Since the approach to equilibrium was made dynamically possible by the definition in the previous section, quasistatic processes are dynamically allowed as well.

Entropy will now be defined by demanding (according to (4) and (5)) that

$$
T \delta S=\delta Q=\delta\langle H\rangle_{\rho_{t}}-\sum_{k}\left\langle\frac{\partial H}{\partial a_{k}}\right\rangle_{\rho_{t}} \delta a_{k}
$$

holds along quasistatic processes in this new understanding. Thus, entropy will be a function of the energy averaged over the ensemble, and the external parameters, just as it was in case of the canonical ensembles. The important difference is that it will now change during processes that are dynamically allowed.

The difficulty that remains is to show that a function $S$ satisfying (10) indeed exists. That is, it has to be shown that an integrating factor exists for $\delta Q$. Remember that entropy is a state function, whereas heat is dependent on the path.

In the appendix, the existence of an entropy function satisfying (10) will be demonstrated for cases in which some assumptions are satisfied, which are reasonable in case the system has mixing dynamics. For this kind of system, a quasistatic process approximates closely a quasi process consisting of consecutive microcanonical distributions. The entropy is shown to be

$$
S=k \ln \Omega\left(\langle H\rangle_{\rho_{t}},\left\{a_{k}\right\}\right)+\text { const. }
$$

which differs from the fine-grained entropy of the microcanonical ensemble only in the argument of $\Omega$. But this is an important difference, since this argument changes in time. Therefore, this entropy function differs from $\mathrm{S}_{f g}$ of the microcanonical ensemble with respect to its evolution over time, but equals it in stationary situations. Hence the "paradox of the constancy of the fine-grained entropy" has been solved.

7. Retrieving the Zeroth and Second Law in Statistical Mechanics. Let me end with a brief discussion of the more general goal of reproducing the laws of thermodynamics. For the zeroth law the prospects are good. In case of mixing dynamics (even only w.r.t. $\Omega$ ) we can immediately 
prove that the system will reach equilibrium, no matter what state it started in, from the definitions in Section 5. If the $\varepsilon_{F}$ are finite, equilibrium will be reached in finite time; if they are zero, it may take an infinite amount of time. For more general systems much work still has to be done, but the fact that we have weakened the standard definition of equilibrium at least guarantees that we're in a good starting position.

So far we have arrived at only part of the second law, namely the equality in (2). To proceed towards the part that concerns nonequilibrium, i.e., the inequality in (2), we could of course invoke Kelvin's principle. But that would mean a retreat to one of the cornerstones of the theory of thermodynamics itself, instead of a reduction of the latter to statistical mechanics. Much progress would be achieved if we could explain the second law from statistical mechanics alone.

Such an explanation is however hard to find. A quotation from Uhlenbeck and Ford 1963 is telling. Rather than giving a derivation of the increase of entropy, they merely express their trust in the credulity of their readers:

I will omit a more detailed discussion about the question in which precise sense the entropy $S \ldots$ has also the property . . . of increasing in any spontaneous transition from one equilibrium state to another. Since we have explained the zeroth law the reader will believe that this part of the second law, which is a more precise expression of the irreversible behaviour of macroscopic systems, can be "explained" in a similar way. $(1963,23)$

Their explanation of the zeroth law, however, only makes it plausible that equilibrium (construed in Boltzmann's manner, as the most probable state) will be reached in the course of time, but does not contain any reference to the entropy of these final equilibrium states. A rigorous explanation of the increase of entropy is therefore still lacking.

The same is true of the account of entropy presented in this paper. Nevertheless some progress has been made: this entropy function has at least the possibility of changing during actual processes.

\section{REFERENCES}

Callender, Craig (1997), Reducing Thermodynamics to Statistical Mechanics: the Case of Entropy. London: LSE Discussion Paper Series (DP 33/97).

Earman, John and Miklós Rédei (1996), "Why ergodic theory does not explain the success of equilibrium statistical mechanics", Brit. J. Phil. Sci. 47: 79 - 91.

Ehrenfest-Afanassjewa, Tatiana (1956), Die Grundlagen der Thermodynamik. Leiden: E. J. Brill.

Gibbs, J. Willard (1902), Elementary Principles in Statistical Mechanics. New Haven: Yale University Press.

Mackey, Michael C. (1993), Time's Arrow: The Origins of Thermodynamic Behavior. New York: Springer. 
Sklar, Lawrence (1993), Physics and Chance. Cambridge: Cambridge University Press.

Uhlenbeck, George E. and George W. Ford (1963), Lectures in Statistical Mechanics. Providence, RI: American Mathematical Society.

\section{Appendix}

We derive expressions for the entropy and temperature, assuming that the system undergoes a quasistatic process, and furthermore obeys

$$
\left\langle\frac{\partial H}{\partial a_{k}}\right\rangle \rho_{t}=\left\langle\frac{\partial H}{\partial a_{k}}\right\rangle_{\mu_{t}} \forall k .
$$

Here $\mu_{t}$ is the microcanonical distribution that is determined by the timedependent parameters $a_{k}(t)$ and $E=\langle H\rangle_{\rho_{t}}$; thus, $\left\{\mu_{t}\right\}$ is a quasi process as discussed in Section 4. The condition (12) can, in view of (9), be fulfilled for mixing systems by making the external changes "infinitely slow."

We first calculate the change of phase space volume as a function of the energy and external parameters:

$$
\begin{aligned}
\delta \Omega\left(E,\left\{a_{k}\right\}\right)= & \int d x\left(\theta\left(E+\delta E-H\left(x,\left\{a_{k}+\delta a_{k}\right\}\right)\right)\right. \\
& \left.-\theta\left(E-H\left(x,\left\{a_{k}\right\}\right)\right)\right) \\
= & \int d x \delta\left(E-H\left(x,\left\{a_{k}\right\}\right)\right)\left(\delta E-\sum_{k} \frac{\partial H\left(x,\left\{a_{k}\right\}\right)}{\partial a_{k}} \delta a_{k}\right) \\
= & \omega\left(E,\left\{a_{k}\right\}\right)\left(\delta E-\sum_{k}\left\langle\frac{\partial H}{\partial a_{k}}\right\rangle_{\mu} \delta a_{k}\right) .
\end{aligned}
$$

(Here $\theta$ is the Heaviside function and the first $\delta$ appearing in the third line is Dirac's delta function.) If we now use the condition (12) and take $\langle H\rangle_{\rho_{t}}$ as the value of the energy in the above expressions, we arrive (using (10)) at the following expression for the amount of heat absorbed by the system during processes which are quasistatic and obey the above condition:

$$
\begin{aligned}
\delta Q & =\delta\langle H\rangle_{\rho_{t}}-\sum_{k}\left\langle\frac{\partial H}{\partial a_{k}}\right\rangle_{\rho_{t}} \delta a_{k} \\
& =\frac{\delta \Omega\left(\langle H\rangle_{\rho_{t}},\left\{a_{k}\right\}\right)}{\omega\left(\langle H\rangle_{\rho_{t}},\left\{a_{k}\right\}\right)} .
\end{aligned}
$$

Hence there exists an integrating factor for these processes. So we identify $T \delta S$ $=\delta Q$, which is satisfied if

$$
\begin{aligned}
T & =\frac{\Omega\left(\langle H\rangle_{\rho_{t}},\left\{a_{k}\right\}\right)}{k \omega\left(\langle H\rangle_{\rho_{t}},\left\{a_{k}\right\}\right)} \\
S & =k \ln \Omega\left(\langle H\rangle_{\rho_{t}},\left\{a_{k}\right\}\right)+\text { const. }
\end{aligned}
$$

(Note that this solution is not unique; the analogue to $S$ could be any function of $\Omega$, and additional arguments are necessary in order to show why the function $k \ln \Omega$ is preferred.) 